DOE/ER/40762-258

UM-PP\#02-058

\title{
Parton Distributions in Light-Cone Gauge: Where Are the Final-State Interactions?
}

\author{
Xiangdong Jif and Feng Yuan门 \\ Department of Physics, University of Maryland, College Park, Maryland 20742
}

(Dated: October 30, 2018)

\begin{abstract}
We show that the final-state interaction effects in the single target spin asymmetry discovered by Brodsky et al. can be reproduced by either a standard light-cone gauge definition of the parton distributions with a prescription of the light-cone singularities consistent with the light-cone gauge link, or a modified light-cone gauge definition with a gauge link involving the gauge potential at the spatial infinity.
\end{abstract}

*Electronic address: xji@physics.umd.edu

${ }^{\dagger}$ Electronic address: fyuan@physics.umd.edu 
Recently, Brodsky and collaborators have re-examined the significance of the parton distributions measurable in deep-inelastic and other high-energy scattering. They found that the final state interactions (FSI) between the struck quark and target spectators yield distinct physical effects such as shadowing and single-spin asymmetry [1, 2]. These effects are of course contained in the light-cone gauge-link explicitly present in the definition of the parton distributions in the non-singular gauges, in which the gauge potential vanishes at the spacetime infinity. In the light-cone gauge, however, the gauge-link vanishes by choice, and the parton distributions in the conventional definition become parton densities which are entirely determined by the ground state light-cone wave functions. Since, Brodsky et al. argue, the wave functions in principle do not encode any information about the FSI, the usual parton distributions in the light-cone gauge must be deficient. When modified to take into account the FSI, they may no longer interpretable as the parton densities. Hence, the provocative claim [1], "structure functions are not parton probabilities."

This of course is not the first time that the light-cone gauge gives us troubles. Over the years, it has been realized that although the light-cone gauge simplifies many calculations significantly, it has associated light-cone singularities at $k^{+}=0$ which are hard to interpret at times. In the earlier days of factorization proofs, it was realized that these singularities prevent contour deformations necessary to cancel certain soft contributions [3]. The factorization theorem for Drell-Yan process, for example, was proved in covariant gauges [4]. In the literature, a number of prescriptions have been suggested to regularize the singularities [5]. Physically light-cone singularities arise from an incomplete gauge fixing for $k^{+}=0$ gluons, corresponding to degrees of freedom in choosing the boundary conditions for the gauge potentials at infinity. Physical results, of course, must be independent of how this additional gauge freedom is being fixed. Nevertheless, in the light-cone gauge, depending on ways residual gauge fixing, certain interesting physics can and do migrate to $k^{+}=0$ or the spacetime infinity. Hence, one must be careful in differentiating interesting physical effects at $k^{+}=0$ such as the FSI from light-cone artifacts.

In this paper, we argue that the standard definition of the parton distributions in the light-cone gauge requires a unique prescription for the light-cone singularities - the one that is constrained to reproduce the light-cone gauge link in the covariant gauge. In this case, the deep-inelastic scaling functions are parton distributions, calculated in a wave function containing the effects of final state interactions! Alternatively, if one demands the initial state wave function be real, then the usual light-cone gauge link in the definition of the parton distributions is incomplete. It ought to be supplemented with an additional contribution. In the non-singular gauges, this new eikonal factor does not contribute. But in the gauges such as the light-cone gauge where the gauge potential does not vanish asymptotically, the additional gauge link is responsible for the final state interactions. We use the example of the single spin asymmetry discussed in Ref. [2] to show that the FSI physics is faithfully reproduced in this approach.

The transverse-momentum parton distribution is defined in the literature as [6]

$$
\begin{aligned}
f\left(x, k_{\perp}\right)= & \frac{1}{2} \int \frac{d \xi^{-} d^{2} \xi_{\perp}}{(2 \pi)^{3}} e^{-i\left(\xi^{-} k^{+}-\vec{\xi}_{\perp} \cdot \vec{k}_{\perp}\right)} \\
& \times\left\langle P\left|\bar{\psi}\left(\xi^{-}, \xi_{\perp}\right) L_{\xi_{\perp}}^{\dagger}\left(\infty, \xi^{-}\right) \gamma^{+} L_{0}(\infty, 0) \psi(0)\right| P\right\rangle,
\end{aligned}
$$

where a suitable regularization is needed for gluon rapidity integrations [7], and $L$ is the 
path-ordered light-cone gauge link

$$
L_{\xi_{\perp}}\left(\infty, \xi^{-}\right)=P \exp \left(-i g \int_{\xi^{-}}^{\infty} A^{+}\left(\xi^{-}, \xi_{\perp}\right) d \xi^{-}\right) .
$$

In hard scattering, the gauge link $L(\infty, 0)$ arises from the final state interactions between the struck quark and the gluon field in the target spectators. Since the quark has extremely high energy and is moving with the speed of light from, say, $z=0$ along the negative $z$ direction, the interaction can be represented by an eikonal line integrating over the whole history of the particle's trajectory, from $t=0$ to $t=\infty$, or $\xi^{-}=0$ to $\xi^{-}=(t-z) / \sqrt{2}=\infty$. In the literature where the factorization theorems are proved, the eikonal line is not considered as final state interactions because the loop integrations over the gluon momenta do not have pinched infrared singularities [8]. As such, one can deform the integrations to a contour on which the momentum exchanges are large, and so the eikonal phase is effectively accumulated almost instantly in the infinite momentum frame. In this paper, however, we follow Ref. [1, 2] by refering the gauge link as (non-pinched) final-state interactions.

In the non-singular gauges, the above definition yields the correct gauge-invariant parton distributions. Indeed the final state interactions are properly represented by the light-cone gauge link [8]. As an example, let us first calculate the asymmetrical part of the transverse momentum distribution in a nucleon due to its transverse polarization, the so-called Sivers function [9, 10]. Since we are only interested in the matter of principle, we use the simple model introduced in Ref. [2] to study the polarization asymmetry discussed there.

Expanding Eq. (1) to the first order in $g$ and dropping the leading term which does not yield any asymmetry in the transverse momentum distribution, we have

$$
\begin{aligned}
f_{1 T}^{\perp}\left(x, k_{\perp}\right)= & \frac{1}{2} \sum_{n} \int \frac{d \xi^{-} d^{2} \xi_{\perp}}{(2 \pi)^{3}} e^{-i\left(\xi^{-} k^{+}-\vec{\xi}_{\perp} \vec{k}_{\perp}\right)}\left\langle P\left|\bar{\psi}\left(\xi^{-}, \xi_{\perp}\right)\right| n\right\rangle \\
& \times\left\langle n\left|\left(-i e_{1} \int_{0}^{\infty} A^{+}\left(\xi^{-}, 0\right) d \xi^{-}\right) \gamma^{+} \psi(0)\right| P\right\rangle+\text { h.c. },
\end{aligned}
$$

where $e_{1}$ is the charge of the struck quark and $n$ represents the intermediate di-quark states. The notation $f_{\perp 1}$ follows Ref. [11] except the kinematic factor is included here. Introducing the interactions between the diquark and gluons, and the nucleon and quark-diquark,

$$
\mathcal{L}_{\text {int }}=-g \bar{\psi} \psi_{N} \phi^{*}-i e_{2} \phi^{*}\left(\overrightarrow{\partial^{\mu}}-\overleftarrow{\partial^{\mu}}\right) \phi A_{\mu}+\text { h.c. }
$$

where $\psi$ represents the quark field, $\psi_{N}$ the nucleon, $\phi$ the charged scalar diquark with charge $e_{2}$. At one-loop order, we have the following expression from Fig. 1,

$$
\begin{aligned}
f_{1 T}^{\perp}\left(x, k_{\perp}\right)= & \frac{-i g^{2} e_{1} e_{2}}{4(2 \pi)^{3} \Lambda\left(k_{\perp}^{2}\right)} \int \frac{d^{4} q}{(2 \pi)^{4}} \bar{U}(P S)(\not k+m) \gamma^{+}(\not k+\not k+m) U(P S) \\
& \times \frac{2(1-x)-q^{+}}{q^{+}+i \epsilon} \frac{1}{(k+q)^{2}-m^{2}+i \epsilon} \frac{1}{(P-k-q)^{2}-\lambda^{2}+i \epsilon} \frac{1}{q^{2}+i \epsilon}+\text { h.c., }
\end{aligned}
$$

where $q^{\mu}$ is the gluon momentum. $M, m$ and $\lambda$ are the masses of the nucleon, quark and diquark, respectively. $U(P S)$ is the on-shell spinor for the nucleon with momentum $P$ and polarization $S . \Lambda\left(k_{\perp}^{2}\right)$ denotes

$$
\Lambda\left(k_{\perp}^{2}\right)=k_{\perp}^{2}+x(1-x)\left(-M^{2}+\frac{m^{2}}{x}+\frac{\lambda^{2}}{1-x}\right) .
$$




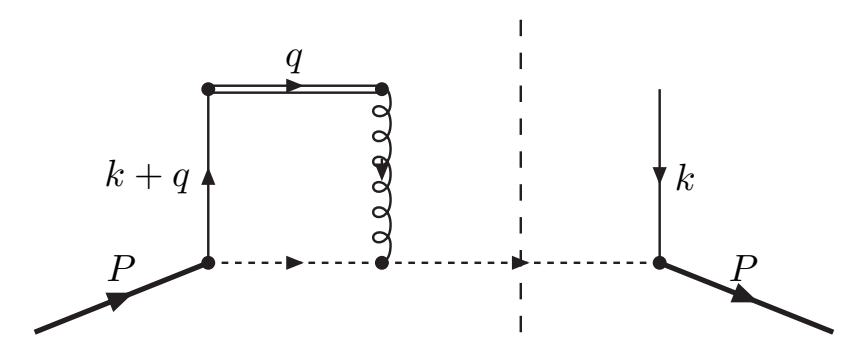

FIG. 1: One-loop contribution to the spin-dependent transverse momentum distribution in the nucleon.

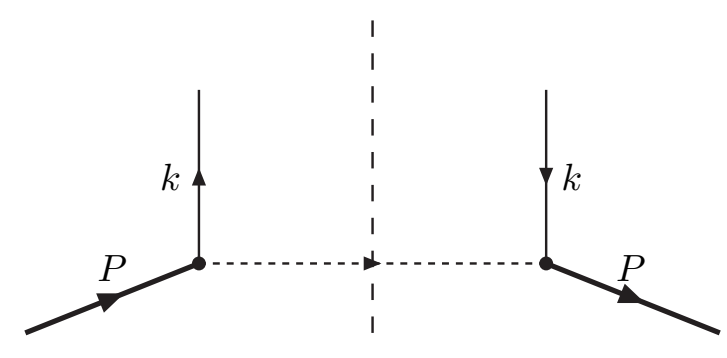

FIG. 2: Tree contribution to the spin-independent transverse momentum distribution.

The $q^{-}$integration can be done by the contour method. Adding the hermitian conjugating contribution results in taking the imaginary part of the eikonal propagator $1 /\left(q^{+}+i \epsilon\right)$. We obtain,

$$
\begin{aligned}
f_{1 T}^{\perp}\left(x, k_{\perp}\right)= & \frac{-i g^{2} e_{1} e_{2}}{8 x(2 \pi)^{3} \Lambda\left(k_{\perp}^{2}\right)}(m+x M) \int \frac{d^{2} q_{\perp}}{(2 \pi)^{2}} \operatorname{Tr}\left[\gamma^{\dagger}\left(\not q_{\perp}-\not k_{\perp}\right) P \gamma_{5} \not s\right] \\
& \times \frac{1}{\left(q_{\perp}-k_{\perp}\right)^{2}} \frac{1}{M^{2}-\frac{q_{\perp}^{2}+\lambda^{2}}{(1-x)}-\frac{q_{\perp}^{2}+m^{2}}{x}} .
\end{aligned}
$$

The integration over $\vec{q}_{\perp}$ yields,

$$
f_{1 T}^{\perp}\left(x, k_{\perp}\right)=\frac{g^{2} e_{1} e_{2}}{(2 \pi)^{4}} \frac{(1-x)(m+x M)}{4 \Lambda\left(k_{\perp}^{2}\right)} \epsilon^{+\alpha \beta \gamma} k_{\perp \alpha} P_{\beta} S_{\gamma} \frac{1}{k_{\perp}^{2}} \ln \frac{\Lambda\left(k_{\perp}^{2}\right)}{\Lambda(0)},
$$

where in the above formulas, we choose $P^{+}=1$.

The spin-independent transverse-momentum distribution has a contribution from the tree diagram shown in Fig. 2. A straightforward calculation leads to

$$
f\left(x, k_{\perp}\right)=\frac{g^{2}(1-x)\left[k_{\perp}^{2}+(x M+m)^{2}\right]}{2(2 \pi)^{3} \Lambda^{2}\left(k_{\perp}^{2}\right)} .
$$

The ratio of the spin-dependent and independent distributions is

$$
\frac{f_{1 T}^{\perp}}{f}\left(x, k_{\perp}\right)=\frac{e_{1} e_{2}(m+x M)}{4 \pi P^{+}} \frac{\Lambda\left(k_{\perp}^{2}\right)}{k_{\perp}^{2}+(x M+m)^{2}} \epsilon^{+\alpha \beta \gamma} k_{\perp \alpha} P_{\beta} S_{\gamma} \frac{1}{k_{\perp}^{2}} \ln \frac{\Lambda\left(k_{\perp}^{2}\right)}{\Lambda(0)} .
$$


This is the same as $\mathcal{P}_{y}$ in Ref. [2] when $S^{\mu}=(0,0,0,1)$. Once again, the calculation demonstrates that the standard definition of the parton distribution in the non-singular gauge does take into account properly the effects of the final-state interactions 8 ].

In the light-cone gauge $A^{+}=0$, however, the gauge link $L$ vanishes. Where are the final state interactions? To find the answer, we consider all contributions to $f\left(x, k_{\perp}\right)$ at one-loop order in both Feynman and light-cone gauges. In the light-cone gauge, the gluon propagator has an extra term

$$
\Delta D^{\mu \nu}(q)=\frac{i}{q^{2}} \frac{q^{\mu} n^{\nu}+q^{\nu} n^{\mu}}{q \cdot n}
$$

where $1 / q \cdot n$ is singular at $q \cdot n=0$ and requires a regularization. In a scattering process, all the contributions coming from this extra term cancel after using the Ward identity following contracting the gluon momentum $q^{\mu}$ with the interaction vertices,

$$
\frac{i}{\not k} \not d \frac{i}{\not k+\not q}=i\left(\frac{i}{\not k}-\frac{i}{\not k+\not q}\right) \text {, }
$$

and the physical amplitude is independent of this extra term (gauge-invariance) and hence the way this singularity gets regularized. An explicit example of this can be found in Ref. [1].

In the case of parton distributions, however, the cancellation is incomplete. Shown in Fig. $3 \mathrm{a}$ are the residual light-cone gauge contributions obtained by contracting $q^{\mu}$ from $\Delta D^{\mu \nu}$ with the vertices, where the shaded lines indicate absence of the propagator after applying the Ward identity. If gauge invariance holds, these contributions must exactly be equal to the corresponding Feynman gauge contributions from the light-cone gauge link shown in Fig. 3b. This happens only when the light-cone gauge singularity is regularized according to the boundary condition imposed on the eikonal link. Therefore, we arrive at an interesting conclusion that the parton distribution in Eq. (1) is valid for the light-cone gauge only when the following light-cone gauge propagator is used

$$
D^{\mu \nu}(q)=\frac{-i}{q^{2}}\left(g^{\mu \nu}-\frac{q^{\mu} n^{\nu}+q^{\nu} n^{\mu}}{q \cdot n+i \epsilon}\right)
$$

where the direction of $q^{\mu}$ is toward the struck quark in its initial state. In other words, one now does not really has a freedom to choose the regularization for the light-cone singularity, contrary to the popular belief. The above propagator can generate phases in the light-cone wave functions, reflecting the effects of the final state interactions, and thus the light-cone wave functions do not just contain the structural information of the initial hadron.

One can of course demand the light-cone wave functions to be real and to contain only information about the initial hadron. This is achieved if we treat $q^{+}$as strictly real and $q^{+}=0$ is approached symmetrically from both sides (principal-value prescription). In this case, we find that the standard light-cone distribution has to be modified in the light-cone gauge, as the gauge potential does not vanish at $\xi^{-}=\infty$. We show that this is the case for the example of single spin asymmetry discussed above.

Consider the Coulomb field generated by a charged particle moving at the speed of light. In the Feynman gauge, one has [12

$$
A^{+}=-\frac{e}{4 \pi} \delta\left(\xi^{-}\right) \ln \mu r_{\perp}, \quad A^{-}=0, \quad A_{\perp}=0 .
$$



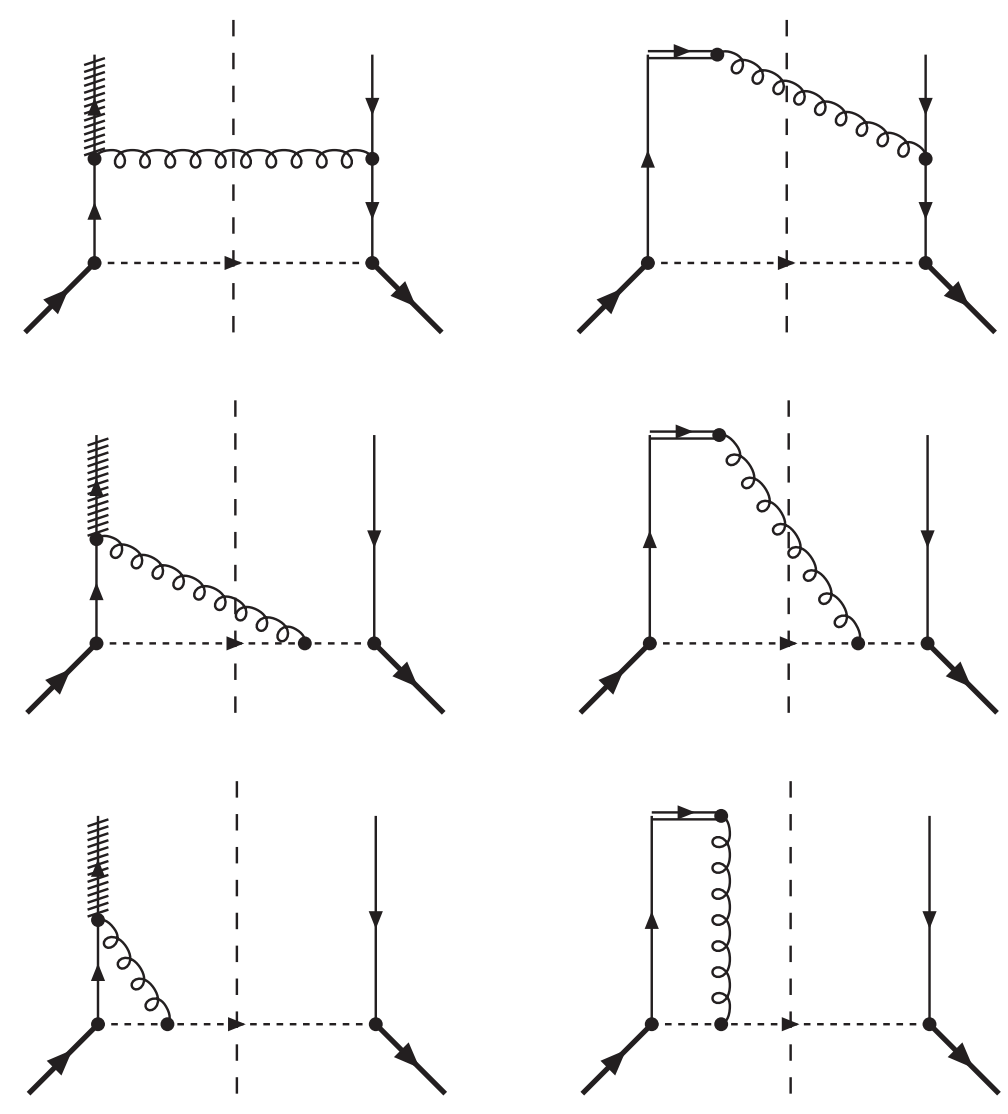

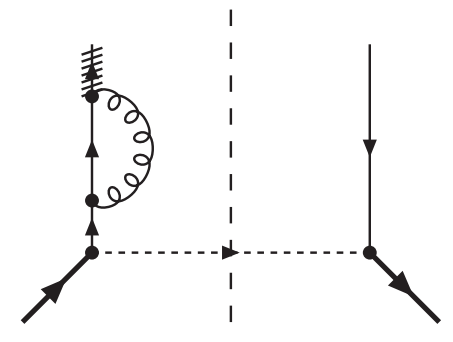

(a)

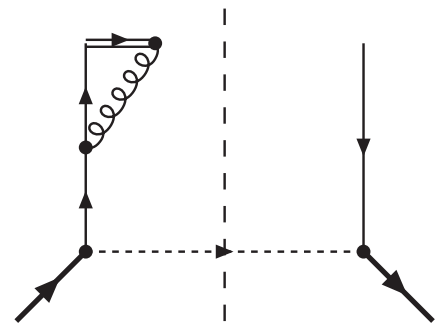

(b)

FIG. 3: a). Extra contributions from the light-cone gauge parton distribution compared to the Feynman gauge distribution without the light-cone link. b) The light-cone-link contributions to the Feynman gauge distribution.

The potential has support only at $\xi^{-}=0$. It has ultra-violet $\left(r_{\perp} \rightarrow 0\right)$ and infrared divergences $\left(r_{\perp} \rightarrow \infty\right)$. The former is related to the physical scale of a probe and the latter is due to the vanishing photon mass. In the light-cone gauge, on the other hand [12],

$$
A^{+}=A^{-}=0, \quad A_{\perp}=-\frac{e}{2 \pi} \theta\left(\xi^{-}\right) \nabla \ln \mu r_{\perp} .
$$

The perpendicular component $A_{\perp}$ does not vanish in the limit $\xi^{-} \rightarrow \infty$. This is the origin of the spurious light-cone singularities. A regularization of the singularities corresponds to a choices of the boundary condition at $\xi^{-}= \pm \infty$. The anti-symmetric boundary condition 
for $A_{\perp}$ corresponds to a principle value regularization [5].

If the gauge potential does not vanish at large $\xi^{-}$, it has a non-vanishing contribution to a gauge link at $\xi^{-}=\infty$. Therefore, the definition of the parton distribution in Eq. (11) is no longer gauge invariant because the two light-cone links generated by $\psi(0)$ and $\psi\left(\xi^{-}, \xi_{\perp}\right)$ are not connected at $\xi^{-}=\infty$. If one makes a gauge transformation $U(\xi)$ which does not vanish at $\xi^{-}=\infty$, an $\mathrm{SU}(3)$ matrix $U^{\dagger}\left(\xi^{-}=\infty, \xi_{\perp}\right) U\left(\xi^{-}=\infty, 0\right)$ pops up in the distribution after the transformation. Therefore, Eq. (1) must be modified to a form that is invariant under a singular gauge transformation.

To motivate a modification, we consider again the motion of the struck quark in deepinelastic scattering. The quark has a large - momentum and thus it travels predominantly along the light-cone $\xi^{-}$direction. However, it also travels along the transverse $\xi_{\perp}$ direction because 1) the quark inherits an intrinsic transverse momentum from the nucleon; 2) it can exchange Coulomb gluons with the target spectators. This transverse motion is justifiably neglected in the eikonal approximation in the nonsingular gauges, and so the accumulation of the eikonal phase is entirely along the $\xi^{-}$direction.

In the light-cone gauge the above approximation is no longer valid. By definition, there is now no phase accumulation along the $\xi^{-}$direction. However, since the phase is physical as it determines the Coulomb scattering cross section, one is forced to conclude that the phase must be accumulated through the motion in the transverse direction. Since the particle is traveling mainly in the $\xi^{-}$direction, the phase accumulation in the transverse direction is very slow; appreciable phase accumulation happens at a very large $\xi^{-}$, or effectively $\xi^{-}=\infty$. Motivated by the above consideration, we modify the eikonal phase in Eq. (1) to,

$$
L_{0}(\infty, 0) \rightarrow \Delta L=P \exp \left(-i g \int_{0}^{\infty} d \xi_{\perp} \cdot A_{\perp}\left(\xi^{-}=\infty, \xi_{\perp}\right)\right),
$$

where the path in the transverse direction is largely arbitrary. It is easy to see that the Coulomb field in the two gauges in Eqs. (11) and (12) yields the same phase factor after integrating over the respective paths.

In the remainder of the paper, we will show that the rescattering effect seen in the covariant gauge is easily reproduced in the light-cone gauge. In fact, consider the parton distribution in Eq. (1) with the gauge link $\Delta L$,

$$
\begin{aligned}
f_{1 T}^{\perp}\left(x, k_{\perp}\right)= & \frac{1}{2} \sum_{n} \int \frac{d \xi^{-} d^{2} \xi_{\perp}}{(2 \pi)^{3}} e^{-i\left(\xi^{-} k^{+}-\vec{\xi}_{\perp} \vec{k}_{\perp}\right)}\left\langle P\left|\bar{\psi}\left(\xi^{-}, \xi_{\perp}\right)\right| n\right\rangle \\
& \times\left\langle n\left|\left(-i e_{1} \int_{\xi_{\perp}}^{\infty} d \xi_{\perp}^{\prime} \cdot A_{\perp}\left(\infty, \xi_{\perp}^{\prime}\right)\right) \gamma^{+} \psi(0)\right| P\right\rangle+\text { h.c. } .
\end{aligned}
$$

Going to the momentum space, we have

$$
\begin{aligned}
f_{1 T}^{\perp}\left(x, k_{\perp}\right)= & \frac{i g^{2} e_{1} e_{2}}{4(2 \pi)^{3} \Lambda\left(k_{\perp}\right)} \int \frac{d^{4} q}{(2 \pi)^{4}} \bar{U}(P S)(\not k+m) \gamma^{+}(\not k+\not h+m) U(P S)\left(2(1-x)-q^{+}\right) \\
& \times \frac{e^{i q^{+} \infty}}{q^{+}} \frac{1}{(k+q)^{2}-m^{2}+i \epsilon} \frac{1}{(P-k-q)^{2}-\lambda^{2}+i \epsilon} \frac{1}{q^{2}+i \epsilon}+\text { h.c. }
\end{aligned}
$$

where $1 / q^{+}$comes from the $n^{-} q^{\perp} / q^{+}$term in the light-cone propagator for the gluon. Using

$$
\lim _{L \rightarrow \infty} \frac{e^{i q^{+} L}}{q^{+}}=i \pi \delta\left(q^{+}\right)
$$


which is true in the sense of principal-valued distribution, we recover the result in Eq. (8).

A consistency check follows when replacing $q^{+}$by $q^{+}+i \epsilon$ in Eq. (19). The exponential factor becomes $\exp (-\epsilon \infty)=0$. Therefore, if Eq. (113) is used from the light-cone gauge propagator, the new gauge link does not contribute. However, the parton distributions defined with the extra gauge link free one from choosing a specific prescription for $1 / q^{+}$. Any prescriptions in fact will yield the same result. In particular, if one uses $q^{+}-i \epsilon$, both the hadron wave function and the extra link contain the effects of the final state interactions.

The gauge link $\Delta L$ can be derived in a similar way as the ordinary light-cone gauge link [13. It does not affect the Dokshitzer-Gribov-Lipatov-Alteralli-Parisi evolution equations of the parton densities in the light-cone gauge because the evolution kernel is real. It is responsible for the shadowing effects (at amplitude level) considered in Ref. [1]. An account of these facts will be published elsewhere [14].

To summarize, we have shown that the final state interactions can be taken into account in the light-cone gauge by either a gauge propagator chosen according to the physics of light-cone gauge link in the usual parton distribution, or an extra gauge link at $\xi^{-}=\infty$ in the parton distribution. In particular, we show that the single spin asymmetry discussed in Ref. [2] is recovered properly in the light-cone gauge.

The authors thank S. Brodsky for a number of useful discussions. We also thank A. Belitsky, J. Collins, P. Hoyer, D. S. Hwang, S. Peigne, and J. W. Qiu for discussions and correspondences. This work was supported by the U. S. Department of Energy via grants DE-FG02-93ER-40762.

[1] S. J. Brodsky, P. Hoyer, N. Marchal, S. Peigne, and F. Sannino, hel-ph/0104291.

[2] S. J. Brodsky, D. S. Hwang, and I. Schmidt, Phys. Lett. B530, 99 (2002).

[3] G. T. Bodwin, S. Brodsky, and G. P. Lepage, Phys. Rev. Lett. 47, 1799 (1981).

[4] G. T. Bodwin, Phys. Rev. D 31, 2616 (1985); D 34, 3932 (1986); J. C. Collins, D. E. Soper, and G. Sterman, Nucl. Phys. B 261104 (1985); B 308, 833 (1988).

[5] A. Bassetto, I. Lazzizzera, and R. Soldati, Nucl. Phys. B 236, 319 (1984); G. P. Lepage and S. J. Brodsky, Phys. Rev. D 22, 2157 (1980); G. Leibbrandt, Rev. Mod. Phys. 59, 1067 (1987); A. A. Slavnov and S. A. Frolov, Theor. Math. Phys. 73, 1158 (1988); Y. V. Kovchegov, Phys. Rev. D 55, 5445 (1997).

[6] J. C. Collins and D. E. Soper, Nucl. Phys. B 194, 445 (1982).

[7] J. C. Collins and D. E. Soper, Nucl. Phys. B 193, 381 (1981). [Erratum-ibid. B 213, 545 (1983).]

[8] J. C. Collins, hep-ph/0204004; also J. C. Collins, Phys. Rev. D 57, 3051 (1998).

[9] D. W. Sivers, Phys. Rev. D 41, 83 (1990); Phys. Rev. D 43, 261 (1991).

[10] M. Anselmino, M. Boglione, and F. Murgia, Phys. Lett. B 362, 164 (1995); M. Anselmino and F. Murgia, Phys. Lett. B 442, 470 (1998); D. Boer and P. J. Mulders, Phys. Rev. D 57, 5780 (1998); D. Boer, Phys. Rev. D 60, 014012 (1999).

[11] P. J. Mulders and R. D. Tangerman, Nucl. Phys. B 461, 197 (1996); Erratum-ibid. 484. 538 (1997).

[12] R. Jackiw, D. Kabat, and M. Ortiz, Phys. Lett. B 277, 148 (1992); I. Robinson and K. Rozga, J. Math. Phys. 25, 499 (1984); G. 't Hooft, Phys. Lett. B 198, 61 (1987).

[13] R. K. Ellis, W. Furmanski, and R. Petronzio, Nucl. Phys. B 212 (1983) 29; J. Qiu, Phys. Rev. 
D 42 (1990) 30.

[14] A. Belitsky, X. Ji and F. Yuan, to be published. 